\title{
25-Hydroxyl Vitamin D Deficiency in Nasal Polyposis
}

\author{
Ankur Kumar Chandrakar ${ }^{1}$ Arun Alexander ${ }^{1}$ () Medha R. $^{2} \quad$ Kalaiselvi Rajendiran ${ }^{2}$ \\ Karthikeyan Ramasamy ${ }^{1}$ \\ ${ }^{1}$ Department of ENT, Jawaharlal Institute of Post Graduate Medical \\ Education and Research, Puducherry, India \\ 2 Department of Biochemistry, Jawaharlal Institute of Post Graduate \\ Medical Education and Research, Puducherry, India \\ Address for correspondence Dr Arun Alexander, MS, DNB, \\ Department of ENT, JIPMER, Puducherry, 605006, India \\ (e-mail: arun.a@jipmer.edu.in). \\ Int Arch Otorhinolaryngol 2020;24(3):e308-e312.
}

\begin{abstract}
Keywords

- Vitamin D

- hs-CRP

- nasal polyposis
\end{abstract}

\section{Introduction}

Nasal polyps are associated with chronic inflammation of the paranasal sinuses and may be associated with atopy. It is still unclear why only some patients with atopy develop polyps. Chronic rhinosinusitis with nasal polyps is a major problem for the patients due to its chronicity, severity of symptoms, recurrence and the unavailability of a standard protocol for its treatment.

The etiopathogenesis of polyps, however, still remains unclear despite advances in molecular biology. ${ }^{1}$ Epithelial cell dysfunction and eosinophilic inflammation of the upper respiratory tract mucosa are other factors that are crucial in the development of nasal polyposis. ${ }^{2}$ There occurs to be an interplay of various inflammatory mediators in the genesis of nasal polyposis. Although nasal polyposis is not a life-threatening condition, it causes significant morbidity interfering with the daily life of the patient and leads to substantial financial burden.

The understanding of various immunomodulatory effects of vitamin $D$ in recent times has encouraged studies for its possible association in the etiopathogenesis of nasal polyposis and received

July 17, 2019

accepted

September 24, 2019

published online

December 13, 2019
DOI https://doi.org/

10.1055/s-0039-3399541. ISSN 1809-9777.
Copyright (e 2020 by Thieme Revinter

Publicações Ltda, Rio de Janeiro, Brazil
License terms

$(\circledast) \Theta \circledast$ 
thereby exploring new targets for intervention. The active form of 25-hydroxyl vitamin D influences innate and adaptive immunity. It acts on antigen-presenting cells and $\mathrm{T}$ cells to promote peripheral tolerance via inhibition of inflammatory responses and induction to T-regs. Vitamin $\mathrm{D}$ is involved in the essential regulatory mechanisms, such as cellular proliferation, differentiation, apoptosis and angiogenesis in various types of cells. ${ }^{3}$ The fact that active Vitamin $D$ has an immunomodulatory role in the human body may make it useful in the treatment of chronic inflammatory diseases like polyposis.

The study on the influence of vitamin D derivatives on the inflammatory process in nasal polyposis may shed light not only on the mechanism of its etiology, but also prove its potential use in the pharmacotherapy of nasal polyposis. Synthesis of vitamin D derivatives of similar anti-proliferative and immunomodulative capabilities without unwanted effects opens new therapeutic challenges. Vitamin D derivatives have been used successfully in the therapy of psoriasis and other hyper-keratotic skin disorders. ${ }^{3}$

C-reactive protein (CRP) is an acute phase reactant, which increases in inflammatory conditions, like nasal polyposis. Its rate of increase seems to be related to the extent of tissue injury and inflammation severity. There are few studies worldwide evaluating the levels of 25-hydroxy vitamin D and high-sensitivity C-reactive protein (hs-CRP) in nasal polyposis.

\section{Aims and Objectives}

The present study assessed the levels of 25-hydroxyl vitamin $\mathrm{D}$ and hs-CRP in patients with nasal polyposis as compared with healthy control subjects and identified their association with disease severity in nasal polyposis. It further assessed the levels of 25-hydroxyl vitamin D and hs-CRP in patients with nasal polyposis and atopy and compared it with patients with nasal polyposis without atopy.

\section{Materials and Methods}

This was a hospital-based cross-sectional study at a major tertiary referral center in India involving two groups - cases and controls. A total of 80 patients with nasal polyposis were recruited from the outpatient clinic of the department of ENT and controls included 80, age- and gender-matched healthy volunteers. Ethical approval was obtained from the Human Ethics Committee of the Institute (JIP/IEC/SC/2014/1/506). Written informed consent was obtained from all study subjects. The study was conducted in accordance with the principles of ethical research as per the Declaration of Helsinki in 1975 and revised in 2008 and the Indian Council of Medical Research Guidelines for Biomedical Research in Human Participants.

\section{Sample Size Calculation}

Sample size was estimated from expected difference in serum hs-CRP values. Previous studies in Japan found the mean difference in hs-CRP levels between subjects and controls to be $14 \mathrm{ng} / \mathrm{ml}$ with a standard difference of $39 .^{5}$ To detect this difference with $80 \%$ power at $5 \%$ level of significance, the minimum sample size required for the study was estimated as 80 in each group (cases and controls). This was calculated using the PS Power and Sample Size Program version 3.0.43 software (Department of Biostatistics, Vanderbilt University, Nashville, Tennessee, USA).

\section{Study Population and Workup}

Subjects with any comorbid condition that could affect vitamin D serum level, such as rickets, osteomalacia, sarcoidosis, thyroid dysfunction, rheumatoid arthritis, ulcerative colitis, Crohn disease, pregnancy, hepatic and renal disease were excluded. Subjects with acute nasal infections and those on any medication for nasal polyposis for the month preceding the workup were also excluded. Adult patients $>18$ years old diagnosed with nasal polyps based on history, examination and radiological investigations were recruited as subjects. In all patients with nasal polyposis, the disease severity was assessed clinically by the Sino nasal outcome test 20 (SNOT20) and radiologically by the Lund \& Mackay staging system. All of the subjects underwent the allergen skin prick test and were divided into atopy or nonatopy subgroups. Common allergens in the community like grass, cereals, common insects and plants were used in the study. A total of 24 different allergens were used to assess atopy. A subject was deemed atopic if he or she was allergic to $>5$ allergens. The association of biochemical parameters with disease severity was also studied.

\section{Assay of Study Parameters}

Biochemical parameters were estimated in the $5-\mathrm{ml}$ blood sample drawn from all study subjects. Levels of 25-hydroxy vitamin D and hs-CRP were measured by enzyme-linked immunosorbent assay (ELISA) using commercially available kits (Quidel Immuno assay, Calbiotech, El Cajon, California, USA). Serum levels of calcium, phosphorus and alkaline phosphatase were also assessed using an auto-analyzer.

\section{Methods of Statistical Analysis}

The normality of continuous data was assessed by the Kolmogrov-Smirnov test. The data was described as mean \pm standard deviation (SD). The comparison of the various parameters between cases and controls was performed by the independent Student $t$-test for parametric data and the Mann-Whitney U-test for nonparametric data. The levels of the various biochemical parameters were correlated with disease severity score between the study parameters and analyzed using the Spearman rank correlation. To assess the effects of confounders, a multivariate linear regression analysis was performed with 25-hydroxyl vitamin $\mathrm{D}$ as a dependent variable. Analysis was performed at the $5 \%$ level of significance and $p<0.05$ was considered statistically significant.

\section{Results}

Eighty patients with nasal polyposis and eighty healthy controls were included in the study. The mean age of onset of nasal polyposis was $37.20 \pm 10.89$ years. The median SNOT score for patients with polyposis was 48 (37-63). The median Lund \& Mackay radiological staging system for patients with polyposis was 11 (2-20). The comparison of baseline characteristics between the 2 groups is shown in - Table 1 . 
Table 1 Comparison of Characteristics Between Cases $(n=80)$ and Controls $(n=80)$

\begin{tabular}{|l|l|l|l|}
\hline Parameter & $\begin{array}{l}\text { Case } \\
\text { Mean } \pm \text { SD }\end{array}$ & $\begin{array}{l}\text { Control } \\
\text { Mean } \pm \text { SD }\end{array}$ & $\begin{array}{l}\boldsymbol{p} \text { value } \\
\text { (Unpaired } t \text {-test) }\end{array}$ \\
\hline Age (years old) & $38.30 \pm 10.92$ & $36.40 \pm 10.61$ & 0.27 \\
\hline Gender (Male:Female) & $48: 32$ & $47: 33$ & - \\
\hline Weekly vitamin D intake IU* & $19.24 \pm 3.56$ & $19.15 \pm 3.65$ & 0.88 \\
\hline Weekly Sunlight exposure (hours) & $8.64 \pm 2.46$ & $9.45 \pm 3.01$ & 0.06 \\
\hline Calcium (mg/dl) & $10.12 \pm 0.41$ & $10.56 \pm 0.48$ & 0.0001 \\
\hline Phosphorus (mg/dl) & $3.97 \pm 0.22$ & $4.02 \pm 0.25$ & 0.53 \\
\hline Alkaline phosphate (IU/L) & $190.00 \pm 17.20$ & $194.40 \pm 16.30$ & 0.41 \\
\hline
\end{tabular}

IIU, International unit. $1 \mathrm{IU}=0.025$ micro gram.

Table 2 Comparison of Study Parameters in Cases $(n=80)$ and Controls $(n=80)$

\begin{tabular}{|l|l|l|l|}
\hline $\begin{array}{l}\text { Study } \\
\text { Parameter }\end{array}$ & $\begin{array}{l}\text { Cases } \\
\text { Mean } \pm \text { SD }\end{array}$ & $\begin{array}{l}\text { Controls } \\
\text { Mean } \pm \text { SD }\end{array}$ & $\begin{array}{l}\text { p-value } \\
\text { (Mann } \\
\text { Witney } \\
\text { U test) }\end{array}$ \\
\hline $\begin{array}{l}25 \text { hydroxy } \\
\text { vitamin D } \\
(\mathrm{ng} / \mathrm{ml})\end{array}$ & $12.01 \pm 7.29$ & $22.87 \pm 14.95$ & $<0.0001$ \\
\hline $\begin{array}{l}\text { hs-CRP } \\
(\mathrm{mg} / \mathrm{L})\end{array}$ & $5.99 \pm 2.74$ & $2.41 \pm 1.95$ & $<0.0001$ \\
\hline
\end{tabular}

Abbreviation: SD, standard deviation.

\section{Vitamin D \& hs-CRP Levels}

Mean 25 hydroxyl vitamin D and hs-CRP levels are shown in - Table 2. Patients in the polyposis group were further subdivided into two subgroups: atopy and non-atopy, based on skin prick test. All of the 80 patients had nasal polyposis, and 65 patients with nasal polyposis had atopy. Our study showed a statistically insignificant comparison of 25-hydroxy vitamin D and hs-CRP between this subgroup.

\section{Correlation Between Severity of Disease and Vitamin D Levels}

There was a strong negative correlation between the vitamin $\mathrm{D}$ levels and hs-CRP in cases. The correlation of 25-hydroxyl vitamin D and hs-CRP levels with symptom severity and radiological scoring was performed in the polyposis group using the Spearman Correlation test. The severity of nasal polyposis was assessed by the SNOT-20 score correlated negatively with serum levels of 25-hydroxyl vitamin $D$ and positively correlated with hs-CRP ( rho $=0.658, p<0.0001$ ).

Similarly, the correlation of the study parameters with radiological disease severity by the Lund-Mackay staging system showed weak negative correlation to vitamin D levels and similar weak positive correlation ( $r h o=0.288, p=0.005$ ) to hs-CRP values.

\section{Discussion}

Nasal polyposis is a chronic inflammatory disease of the nose and paranasal sinuses. Several factors may play a role in the etiopathogenesis of nasal polyposis, including genetic predisposition, infection, anatomic variations, allergy, and immunological disorders, although this may not be clearly demonstrated in all aspects. The histological basis of nasal polyposis includes presence of tissue eosinophilia and secretion of the related inflammatory mediators. ${ }^{1,2}$

The present study was planned to address the question of the interplay between systemic inflammation and serum 25-hydroxyl vitamin D levels in patients with nasal polyposis. The study was addressed to identify differences in these parameters in nasal polyposis patients of varying severity with age- and gender-matched healthy volunteers. Moreover, those with other causes of inflammation, with any comorbidity or on any medication including vitamin D supplementation were excluded. The disease severity score was recorded by a single investigator, avoiding variability.

There has been plenty of debate on the definition of Vitamin D deficiency. The most acceptable definition is serum level of 25-hydroxyl vitamin $\mathrm{D}<20 \mathrm{ng} / \mathrm{mL}$ (or $50 \mathrm{nmol} / \mathrm{L}$ ), which is recognized as a pathological condition characterized by muscle weakness, rickets or osteomalacia. Whereas serum levels of 25-hydroxyl vitamin D ranging from 10 to $30 \mathrm{ng} / \mathrm{mL}$, without overt clinical symptoms is defined as Vitamin D insufficiency. This has recently become an important medical concern. ${ }^{3,4}$ Substantial evidence suggests that vitamin D plays a major role in immunomodulation because Vitamin D receptors are present on several types of immune competent cells, including T cells, B cells, macrophages and dendritic cells. ${ }^{5}$

There is more evidence in various studies about the immune regulatory functions of vitamin $\mathrm{D}$ in allergic diseases such as asthma and allergic rhinitis. Vitamin D can reduce the level of cytokines released by the inflammatory cells. ${ }^{6}$ The strong correlation between Vitamin D deficiency and hs-CRP levels seen in the present study demonstrates the strong link between Vitamin D and its anti-inflammatory properties.

We investigated the presence of a possible link between vitamin D deficiency and nasal polyposis in the Indian population, as there were no previous studies cited in literature. Our study showed a significant low level of vitamin D in the case group as compared with the controls. The normal level of serum vitamin D is $>30 \mathrm{ng} / \mathrm{ml}$. We observed that the level of vitamin D was low in both the case and control groups, but the 
average level of vitamin D in the case group was almost half when compared with the control group.

In a study conducted by Sule et $\mathrm{al}^{7}$ on 60 adult patients having nasal polyposis with or without allergic rhinitis and 40 healthy controls to evaluate the relationship between Th1/Th2 cell balance by measuring the levels of IL-4, IL-10, IFN- $\Upsilon$ and $1 \alpha, 25$-dihydroxyvitamin D3, they observed that the serum level of 25-OH vitamin D was significantly lower in nasal polyposis patients with allergic rhinitis than in the control group $(p<0.05)$, while there was no significant difference in serum level of $25-\mathrm{OH}$ vitamin $\mathrm{D}$ between nasal polyposis without allergic rhinitis and the control group $(p>0.05))^{7}$

Saba et $\mathrm{al}^{6}$ measured the vitamin D levels in patients with allergic rhinitis and study results were compared with the general population of Iran. They found that prevalence of vitamin D deficiency was significantly higher in patients with allergic rhinitis (30\%) than in the normal population $(5.1 \%)^{7}$. Wjst et al, in their study of 18,224 adults, indicated an increase in the prevalence of allergic rhinitis in vitamin D deficient subjects. ${ }^{8}$

Our study failed to establish a statistically significant difference $(p=0.261)$ between the vitamin D serum levels of patients with nasal polyposis having allergic rhinitis and the patients with nasal polyposis without allergic rhinitis. This was possibly because of the inadequate patient distribution in both subgroups. This was a limitation of the present study. This observation is similar to the study conducted by Sule et al where no statistical significance was found between vitamin $\mathrm{D}$ levels in patients with nasal polyposis without allergic rhinitis and in the control group of healthy subjects $(p>0.05) .^{7}$

To our knowledge, there is no literature evaluating the relationship between vitamin $\mathrm{D}$ levels and severity of nasal polyposis assessed by clinical and radiological scales. Our study showed the median value of SNOT score was 48 (range 37-63) in the case group. This corresponds to a moderate to severe nasal polyposis disease. We found the median value of the Lund-Mackay score 11 (range 2-20) in the case group. This corresponds to a mild to moderate type of disease. In our study, the levels of vitamin $\mathrm{D}$ demonstrated a negative correlation with disease severity by the SNOT score and the Lund and Mackay staging system.

Mulligan et al showed a strong inverse relationship between reduced plasma levels of vitamin $\mathrm{D}$ and an increase in the CT (Computed tomography) bone remodeling score in chronic rhinosinusitis $(p=0.009)$. The average CT bone remodeling score in patients with insufficient levels $(<32 \mathrm{ng} / \mathrm{ml})$ of serum vitamin $\mathrm{D}$ was significantly greater than in patients with adequate $(>32 \mathrm{ng} / \mathrm{ml}$ ) vitamin $\mathrm{D}$ levels $(p=0.016)$. This result supports the role of vitamin $\mathrm{D}$ in the exacerbation of chronic rhinosinusitis-associated bone erosion. ${ }^{9}$

C-reactive protein is an acute phase reactant and its level increases in inflammatory conditions like nasal polyposis. Acute phase proteins are systemic markers of inflammatory response that are used in the diagnosis and prognosis of the course of the inflammatory process. Recent studies show the role of CRP in immunity. C-reactive protein levels are signifi- cantly higher in patients with polyps, suggesting systemic inflammation in nasal polyposis. We observed in our study that the serum hs-CRP levels were elevated in nasal polyposis patients and increased linearly with increasing disease severity. To our knowledge, there is no literature evaluating the relationship between concentration of serum hs-CRP and disease severity of nasal polyposis. We observed a statistically significant moderately positive correlation between the levels of hs-CRP and the SNOT score $(p<0.0001)$ and a weak positive correlation between hs-CRP and the Mackay- Lund staging system $(p=0.005)$. We can conclude that a patient with severe nasal polyposis had a higher level of CRP and corresponded to a higher disease severity score.

Partyka et al studied the usefulness of the hs-CRP and ferritin assay in 38 postoperative patients of nasal polyposis and found a statistical difference in the results. Hence, the authors concluded that the levels of CRP and ferritin can be used in early postoperative detection of inflammatory state in patients with nasal polyposis and for the effectiveness of the therapy. ${ }^{10}$ Yildirim et al measured high sensitivity CRP levels in 100 patients divided into 4 equal number of groups, namely: patient with allergic rhinitis, CRS (Chronic rhino-sinusitis) with nasal polyposis, CRS without nasal polyposis, and a nonsmoker healthy control group. They found no statistically significant difference in the hs-CRP levels between individuals in the first 3 groups and in those of the control group. ${ }^{11}$ Büyüköztürk et al studied acute phase reactants (CRP, serum amyloid A [SAA] and fibrinogen) in allergic rhinitis and asthma and demonstrated that acute phase reactant SAA rises in patients with allergic rhinitis and in patients with asthma, but the CRP and fibrinogen levels found were statistically insignificant in allergic airway disease when compared with the control group. ${ }^{12}$

We observed that there was significant systemic inflammation and lowered 25-OH vitamin levels in patients with nasal polyposis, as these markers were found to correlate with the disease severity score of nasal polyposis. It might be possible for the physician to use them to monitor therapeutic response. We can conclude that vitamin D supplementation might reduce systemic inflammation. More longitudinal studies are needed to assess this in the future.

\section{Limitations of the Study}

1. Computed tomography (CT) was used to assess the disease severity of the cases of nasal polyposis. However, it is a protocol that patients requiring $\mathrm{CT}$ scan are advised CT only after a course of oral and topical steroids, and CT is then requested if the patients show no improvement. Thus, the true extent of the disease is not accurately assessed, meaning that the results of correlation with vitamin D levels are possibly erroneous. It is not practical to get a CT at the first sitting and one at a later date due to the unnecessary radiation exposure of multiple CT scans.

2. Our study found an overwhelming number of patients with polyps having associated atopy, as expected in the literature. However, this resulted in a small sample size for the group of patients with polyps and without associated atopy. Subgroup analysis in this group (with polyp and 
without atopy) for correlation between vitamin D levels and hs-CRP or disease severity was not adequately powered and, hence, future studies are required with a larger sample size to explore this association. It is only then that some light can be shed on why polyps develop in some patients even without allergy.

\section{Conclusion}

Nasal polyps are associated with chronic inflammation of the paranasal sinuses and mainly associated with atopy. Vitamin $\mathrm{D}$ deficiency is common in the Indian population and deficiency of vitamin D leads to significant inflammation and leads to polyps.

The study has shown a significant correlation between vitamin $\mathrm{D}$ deficiency and inflammation in patients with nasal polyps. This study has shown that disease severity has some relation to vitamin D levels, although not as well as its relation to paranasal sinus involvement on CT scanning.

From our study, we can see a possible role of active vitamin D and its analogues supplementation in the prevention and control of nasal polyposis. Synthesis of vitamin D derivatives of similar antiproliferative and immunomodulative capabilities may open new therapeutic possibilities in the future.

\section{Funding}

JIPMER INTRAMURAL FUND, (Grant / Award Number: 'JIP/Res/Intra-MD/MS/01/2014')

\section{Conflicts of Interests}

Dr. Alexander reports grants from JIPMER, during the conduct of the study.

\section{References}

1 Pawankar R. Nasal polyposis: an update: editorial review. Curr Opin Allergy Clin Immunol 2003;3(01):1-6

2 Fan G-K, Wang H, Takenaka H. Eosinophil infiltration and activation in nasal polyposis. Acta Otolaryngol 2007;127(05):521-526

3 Cunliffe WJ, Berth-Jones J, Claudy A, et al. Comparative study of calcipotriol (MC 903) ointment and betamethasone 17-valerate ointment in patients with psoriasis vulgaris. J Am Acad Dermatol 1992;26(5 Pt 1):736-743

4 Johnson CS, Hershberger PA, Trump DL. Vitamin D-related therapies in prostate cancer. Cancer Metastasis Rev 2002;21(02):147-158

5 Hackstein H, Thomson AW. Dendritic cells: emerging pharmacological targets of immunosuppressive drugs. Nat Rev Immunol 2004;4(01):24-34

6 Arshi Saba Ghalehbaghi B, Kamrava SK, Aminlou M. Vitamin D serum levels in allergic rhinitis: any difference from normal population? Asia Pac Allergy 2012;2(01):45-48

7 Ozkara Sule, Keles E, Ilhan N, Gungor H, Kaygusuz I, Alpay HC. The relationship between Th1/Th2 balance and $1 \alpha, 25$-dihydroxyvitamin $\mathrm{D}_{3}$ in patients with nasal polyposis. Eur Arch Otorhinolaryngol 2012;269(12):2519-2524

8 Wjst M, Hyppönen E. Vitamin D serum levels and allergic rhinitis. Allergy 2007;62(09):1085-1086

9 Mulligan JK, Bleier BS, O'Connell B, Mulligan RM, Wagner C, Schlosser RJ. Vitamin D3 correlates inversely with systemic dendritic cell numbers and bone erosion in chronic rhinosinusitis with nasal polyps and allergic fungal rhinosinusitis. Clin Exp Immunol 2011;164(03):312-320

10 Partyka R, Pałac J, Paluch Z, et al. Evaluation of usefulness of hs-CRP and ferritin assays in patients with nasal polyps. Dis Markers 2014; 2014:794060

11 Yıldırım YS, Apuhan T, Koçoğlu E, Simşek T, Kazaz H. High sensitivity C-reactive protein levels in chronic rhinosinusitis and allergic rhinitis. Kulak Burun Bogaz Ihtis Derg 2011;21(05): 266-269

12 Büyüköztürk S, Gelincik AA, Genç S, et al. Acute phase reactants in allergic airway disease. Tohoku J Exp Med 2004;204(03):209-213 